\title{
The Influence of Nd: YAG Laser Energy on Plasma Characteristics Produced on Si: Al Alloy Target in Atmosphere Pressure
}

\section{Hamid H Murbat ${ }^{1^{*}}$ and Husham Abdul Hamza ${ }^{2}$}

${ }^{1}$ Department of physics, College of science for woman, University of Baghdad, Iraq

${ }^{2}$ Department of physics, College of science, University of Baghdad, Iraq

*Corresponding author: Hamid H Murbat, Department of physics, College of science for woman, University of Baghdad, Iraq, E-mail: hamidmurbat@gmail.com

Received Date: June 20, 2017; Accepted Date: July 17, 2017; Published Date: July 19, 2017

Citation: Hamid H Murbat, et al. (2017) The Influence of Nd: YAG Laser Energy on Plasma Characteristics Produced on Si: Al Alloy Target in Atmosphere Pressure. J Mater sci Appl 3: 1-7.

\begin{abstract}
Background: The spectroscopy study of atomic emission from the expanding plasma plume formed during the laser-matter interaction what is called Laser Induced Breakdown Spectroscopy (LIBS). It provides valuable information about the composition of the target material. In this study, The plasma produced by the interaction of high fluence Nd: YAG laser at the wavelength of $1064 \mathrm{~nm}$ with Silicon-Aluminum target (Si:Al). Studied the laser-induced plasma generated at different laser energies has been characterized using optical emission spectroscopy. High power pulse laser is used to generate plasma from (Si:Al) target. The electron temperatures $\left(\mathrm{T}_{\mathrm{e}}\right)$ and plasma density $\left(\mathrm{n}_{\mathrm{e}}\right)$ were calculated by Boltzmann plot method from singly ionized aluminum emission lines (Al II), and Saha equation, respectively. $\mathrm{T}_{\mathrm{e}}$ and $\mathrm{n}_{\mathrm{e}}$ utilized to evaluate the plasma parameters such as Debye length $\left(\lambda_{D}\right)$, number of particles in Debye sphere $\left(N_{D}\right)$ and plasma frequency $\left(\omega_{p}\right)$.
\end{abstract}

Keywords: Laser produced plasma; Nd: YAG laser; Si:Ai alloy; Emission spectroscopy

\section{Introduction}

Plasmas are not often observed naturally on earth with the exception of lightning or the aurora borealis. Plasmas appear in space and astrophysics, laser-matter interaction, technology, fusion, etc. Technical plasmas, magnetic fusion plasmas and laser-generated plasmas are the main applications of plasma physics on earth [1].

In especially laser plasmas have extreme properties not found in more conventional plasmas (e.g., densities characteristic of solids). Fusion energy known as inertial confinement fusion is a major application of laser plasma physics, in this approach, high energy laser beams were focused towards to a small solid target to explode its until the densities and temperatures characteristic reaches to nuclear fusion. Another field of using laser plasma physics was in the particle accelerators, where a extremely strong electric fields was generated when a high intensity laser pulse passes through plasma to accelerate particles. High-energy physicists hope to reduce the size and cost of particle accelerators by using plasma acceleration techniques [2].
An interesting application in the field of laser induced plasma is Laser Induced breakdown spectroscopy (LIBS). LIBS is an atomic emission spectroscopy technique which utilizes a laser pulse with sufficient high energy as excitation source. LIBS can be used to analyze any state of matter (solid, liquid or gas). Almost all elements can be detected by LIBS because of thy emit light when excited to sufficiently high energies, depends only on the laser power as well as on the detector sensitivity of the dispersed radiation and wavelength range of the spectrometer [3]. Atomic emission spectroscopy is a well-established technique for the measurement of materials. Excitation of atoms may be performed by raising the temperature of the sample using a flame [4], an inductively coupled plasma [5], a microwave induced plasma, an arc or spark between electrodes [6-7]. Emission measurements are preferred over absorption measurements when high sensitivity is required. An absorption signal is measured as the reduction in transmitted signal, "negative signal", in which small absorptions involves detection of small signal decrease on a large transmitted background. However, emission signal is zero for no signal, then emission measurements can be performed with a better sensitivity than absorption measurements. 
A large number of studies have been made on the laser induced plasma on solid, liquid surfaces and in a gas. The plasma is produced by focusing a laser beam in test sample.

The aim of our study is to study the physical properties of the laser induced plasma in water. One of application of this study is to improve the quality of water by resolving the environmental pollutants [8].

\section{Optical Emission Spectroscopy}

Optical Emission Spectroscopy (OES) is broadly used technique, mainly because it is easy to implement and non-intrusive. It also relies on the study of the shape and width of spectral lines that can be altered by numerous mechanisms.

The electron temperature of plasma was calculated using Boltzmann relation (1):

$$
\operatorname{Ln}\left(\frac{I_{m n} \lambda_{m n}}{h c g_{m} A_{m n}}\right)\left(-\frac{E_{m}}{K_{\beta} T_{e}}\right)+C
$$

where $\lambda \mathrm{mn}, \mathrm{Imn}, \mathrm{gm}$, and Amn are the wavelength, intensity, statistical weight, and transition probability between the transition states of upper level (m) and lower level (n), respectively, and $\mathrm{C}$ is constant [9].

The electron density was calculated using Saha-Boltzmann equation (2) for atom and ion spectral lines emitted from the plasma:

$$
n_{e}=\frac{I_{z}}{I_{z+1}^{*}} 6.04 \times 10^{21}(T)^{3 / 2} e \frac{E_{j, Z+1}-\chi_{z}}{k T}
$$

Where $I_{z}^{*}=I_{z} \lambda_{j i, z} / g_{j, z} A_{j i, z}, \chi$, is the ionization energy of the species in ionization stage $\mathrm{z}$ in $\mathrm{eV}, \mathrm{Tz}$ is the line intensity for transition from upper level-j to lower level-i, $\lambda_{j i, z}$ is the corresponding wavelength of transition from level-j to level-i, is the statistical weight of transition from level-j, $A_{j i, z}$ is the transition probability from level-j to level-i and $\mathrm{T}$ is the electron temperature [9].

Other fundamental plasma parameter such as Debye length $\left(\lambda_{D}\right)$, plasma frequency of electron $\left(\omega_{P}\right)$ and Debye number $\left(N_{D}\right)$ can be calculate by the following equations:

$$
\lambda_{D}=\left(\varepsilon_{0} K_{\beta} T_{e} / n_{e} e^{2}\right)^{1 / 2}=7.43 \times 10^{2} \sqrt{T_{e} / n_{e}}
$$

$\omega_{p e}=\sqrt{\frac{e^{2} n_{e}}{\varepsilon_{0} m_{e}}}=2 \pi f_{p}$

$$
N_{D}=\frac{4}{3} \pi n\left(\lambda_{D}\right)^{3}=1.38 \times 10^{6}(T)^{3 / 2} / n^{1 / 2}
$$

\section{$\left(\operatorname{Tin} K^{\circ}\right)$}

Where is permittivity of free space, $\kappa_{\beta}$ the Boltzmann constant, e the electron charge, $\eta_{e}$ is electron density and $m_{e}$ is electron mass.

\section{Experimental Setup}

Nd: YAG laser (Hua Fei Tong Da Technology-Diamond -288 Pattern EPLS) with different peak powers in air on target, was used in optical emission spectroscopy of laser induced plasma, with following characteristics:

- Laser model: Q-switched Nd: YAG laser first harmonic generation.

- Laser peak power: (6- 36) MW.

- Laser wavelength: (1064) nm.

- Repetition rate: (1- 10) Hz.

- Cooling method: inner circulation water cooling.

- Input Ac power supply: (220) Volt.

The laser spot diameter can be changed by changing the distance between the laser lens and the target. Pulse duration $(9 \mathrm{~ns})$ with $6 \mathrm{~Hz}$ repetition frequency. The distance fixed during the measurements for system accuracy and precision. In this work, we used $10 \mathrm{~cm}$ focal length lens. A shorter focal length lens can produce a small beam waist, and therefore, stronger breakdown, but it also has a smaller depth of focus, Figure 1 shows a schematic diagram for the LIBS setup (1).

The spectrometer analysis was done using the light emitted from sample bombarded by the pulse laser. The spectrometer with short response time from Ocean Optics (HR 4000 CG-UV-NIR) was used in the setup to analyze emitted light.

The light produced by the ablated plasma was collected by the optical fiber which was set at angle of about 45 degree to axes of the laser beam to avoid splashing and then guided to the entrance slit of the spectrometer. The spectrometer has a high resolution depending on grating used in it, and responds to a wavelength between (200-900) nm with 3648 pixels. Nd: YAG laser at $1064 \mathrm{~nm}$ is tightly focused on the target to produce plasma plume. 
In order to insure exposing a fresh surface after every train of shots the target surface was rotated manually. The laser pulse energy was varied from 300 to $800 \mathrm{~mJ}$, each spectrum was obtained over a (200-700) nm wavelength range.

Finally the results were analyzed and compared with National Institute of Standards and Technology data (NIST) [10] and evaluate the plasma parameters such as electron density (ne), electron temperature (Te), plasma frequency (fp), Debye length $(\lambda D)$ and number of particles in Debye sphere (ND) at different laser energy.

\section{Results and Discussion}

Light emitted from the plasma is detected and the resulting spectrum distribution plotted as intensity in arbitrary units against wavelength $(\mathrm{nm})$.

The optical emission spectra of Si: Al alloy target plasma which confined in atmosphere was recorded using (OES) technique. Figure 2 shows the emission spectra of laser induced on Si: Al alloy target in air in the spectral range 225-640 $\mathrm{nm}$.

It is clear from this Figure that the intensity of the spectral lines increase with the increase in the laser peak power from 300 to $800 \mathrm{~mJ}$, this can interpreted as follows:

The increase of laser power leads to mass ablation rate from the target, means more excited atoms and hence increasing in the height of the spectral line intensities. The increase in laser power will increase its absorption in the plasma resulting in more ablation as well as plasma emission.

The prominent Silicon-Aluminum (Si: Al) alloy target in air in spectral lines observed in Al I (237.3124, 257.5393, 309.27099 and 396.152) $\mathrm{nm}$. Through the use of these wavelengths plasma parameters were calculated.
The electron temperature $\left(\mathrm{T}_{\mathrm{e}}\right)$ was determine from the slope of the best linear fit in Boltzmann plot (against upper level energy (for corresponding transition) Boltzmann plot requires peaks for the same atomic species and the same ionization state (Al $\mathrm{I}$ in this part). Figure 3 shows the best linear fit in Boltzmann equation for different laser energies.

The obtained results show that increases laser pulse energy, leads to increase in the electron temperature, Debye length and Debye number, while the electron density and plasma frequency were decreased as shown in Figure 4.

Table 1 display the calculated electron density (ne), electron temperature (Te ), plasma frequency ( $\mathrm{fp}$ ), Debye length $(\lambda D)$, ) and Debye number (ND) for Si: Al target at different laser pulse energies. All calculated plasma parameters $(\lambda D, f p$ and $N D)$ were satisfied the criteria for the plasma. It shows that fp decrease with laser energy because it is proportional with ne, while $\lambda \mathrm{D}$ and $\mathrm{Nd}$ increase with it such as in Ref [11].

\section{Conclusions}

1. The spectral lines intensities of the laser induced plasma emission exhibited a strong dependence on the ambient conditions. It is found that the intensities at different laser peak powers increase with increasing laser peak power and then decreases when the power continues to increase.

2. Plasma parameters such as electron temperature, electron density, Debye length, number of particles in Debye sphere and plasma frequency are found to be strongly influenced by the laser energy.

3. The results showed that the values of $T_{e}, N_{D}$ and $\lambda_{D}$ were increased in case of laser induced plasma in air environment while the values of $n_{e}$ and $f_{p}$ were decreased in laser induced plasma air environment.

\begin{tabular}{|l|l|l|l|l|l|}
\hline Laser energy $(\mathrm{mJ})$ & $\mathrm{Te}(\mathrm{eV})$ & $\mathrm{ne} 1016(\mathrm{~cm}-3)$ & $\mathrm{fp}(\mathrm{Hz})^{\star} 1012$ & $\lambda \mathrm{D}{ }^{\star} 10-5(\mathrm{~cm})$ & $\mathrm{ND}$ \\
\hline 300 & 0.833 & 34.308 & 5.260 & 0.116 & 2.231 \\
\hline 400 & 0.914 & 33.720 & 5.215 & 0.122 & 2.586 \\
\hline 500 & 1.080 & 32.480 & 5.118 & 0.135 & 3.383 \\
\hline 600 & 1.157 & 31.708 & 5.057 & 0.142 & 3.796 \\
\hline 800 & 1.199 & 26.758 & 4.645 & 0.157 & 4.362 \\
\hline
\end{tabular}

Table 1: plasma parameters for Si: $\mathrm{Al}$ in air with different laser energy 


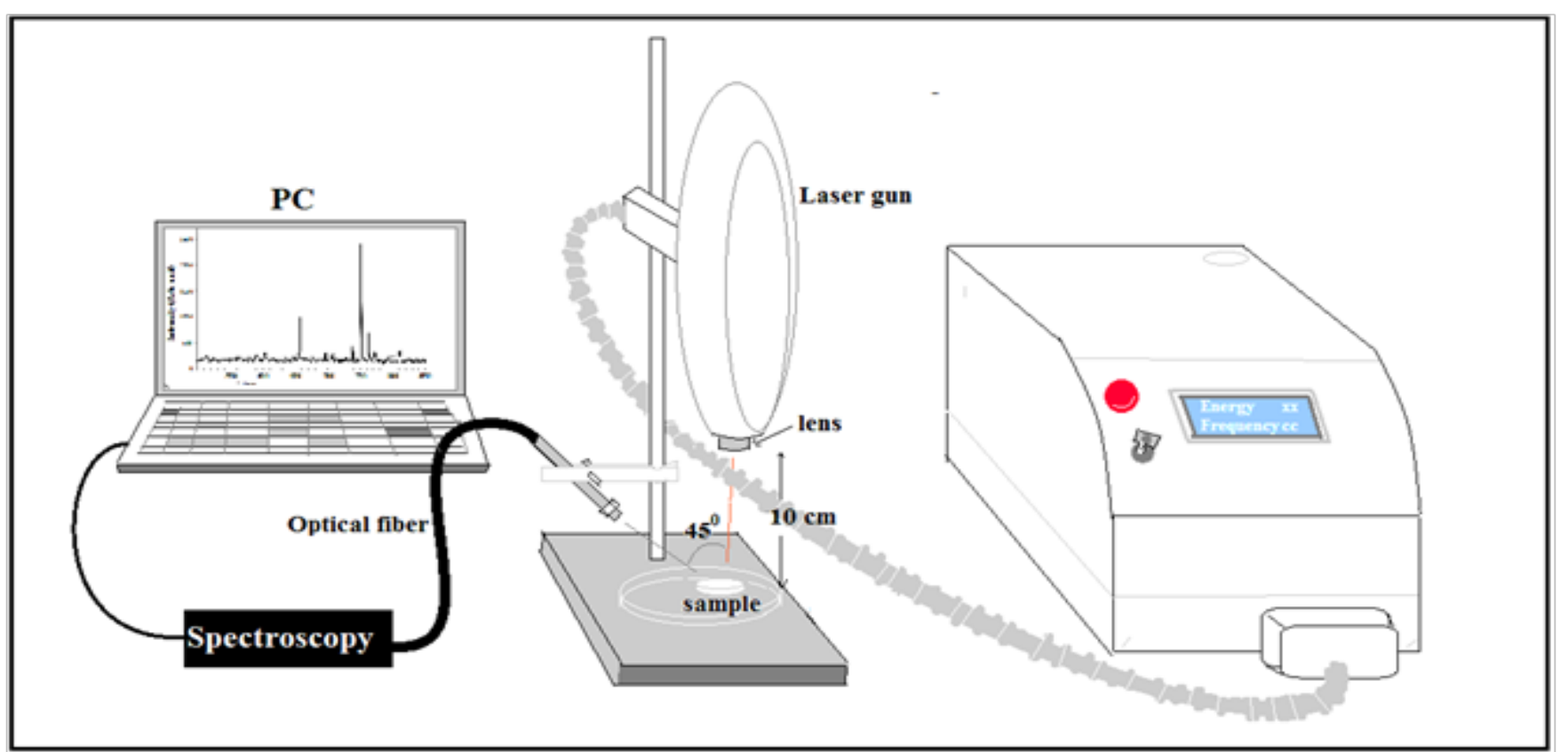

Figure 1: Laser Induced Plasma Spectroscopy (LIBS) system configuration

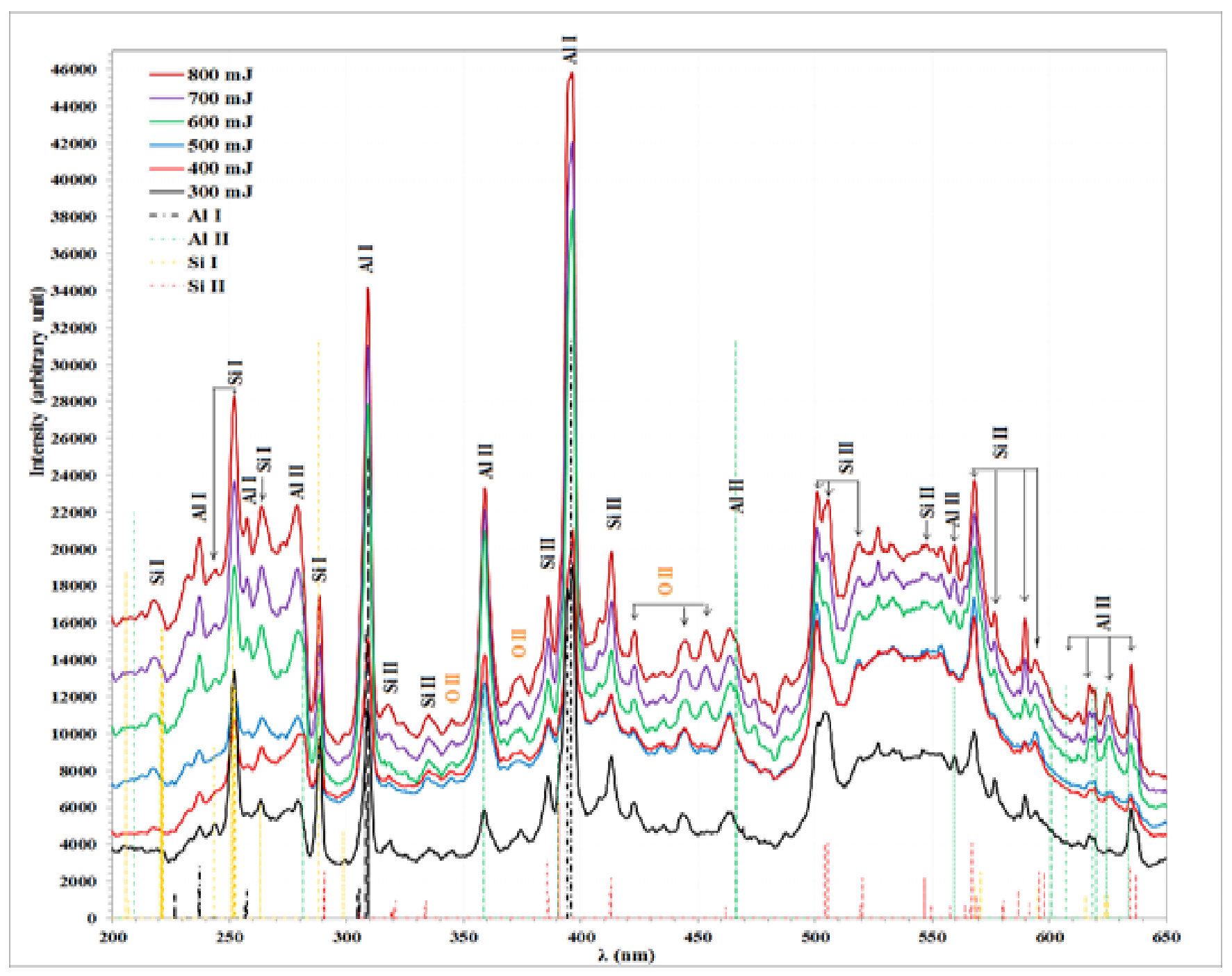

Figure 2: Emission spectra of laser induced on Si-Al alloy target in air with different laser energies 

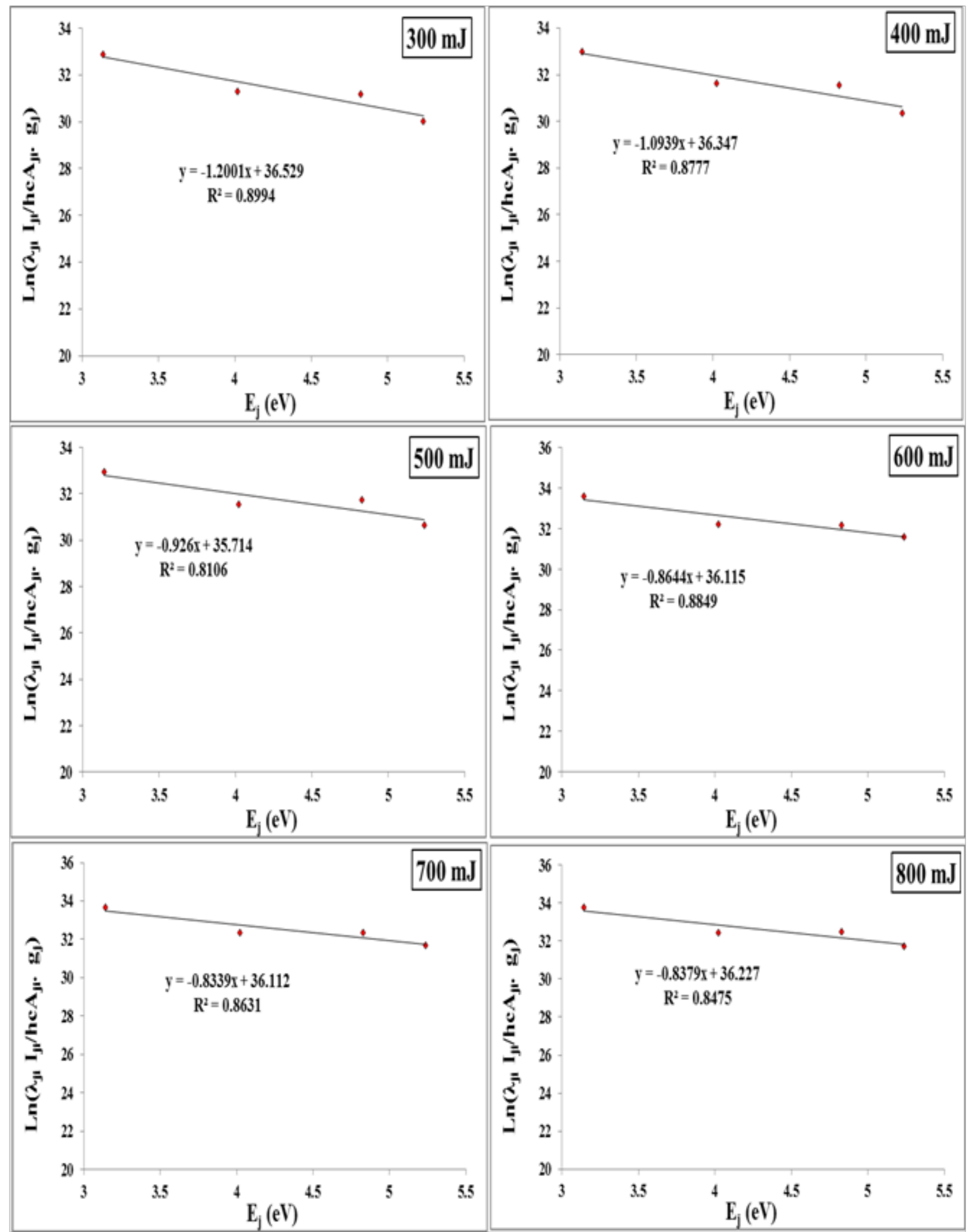

Figure 3: Boltzman plot for plasma produced on Si: Al alloy target in distilled water with different laser 


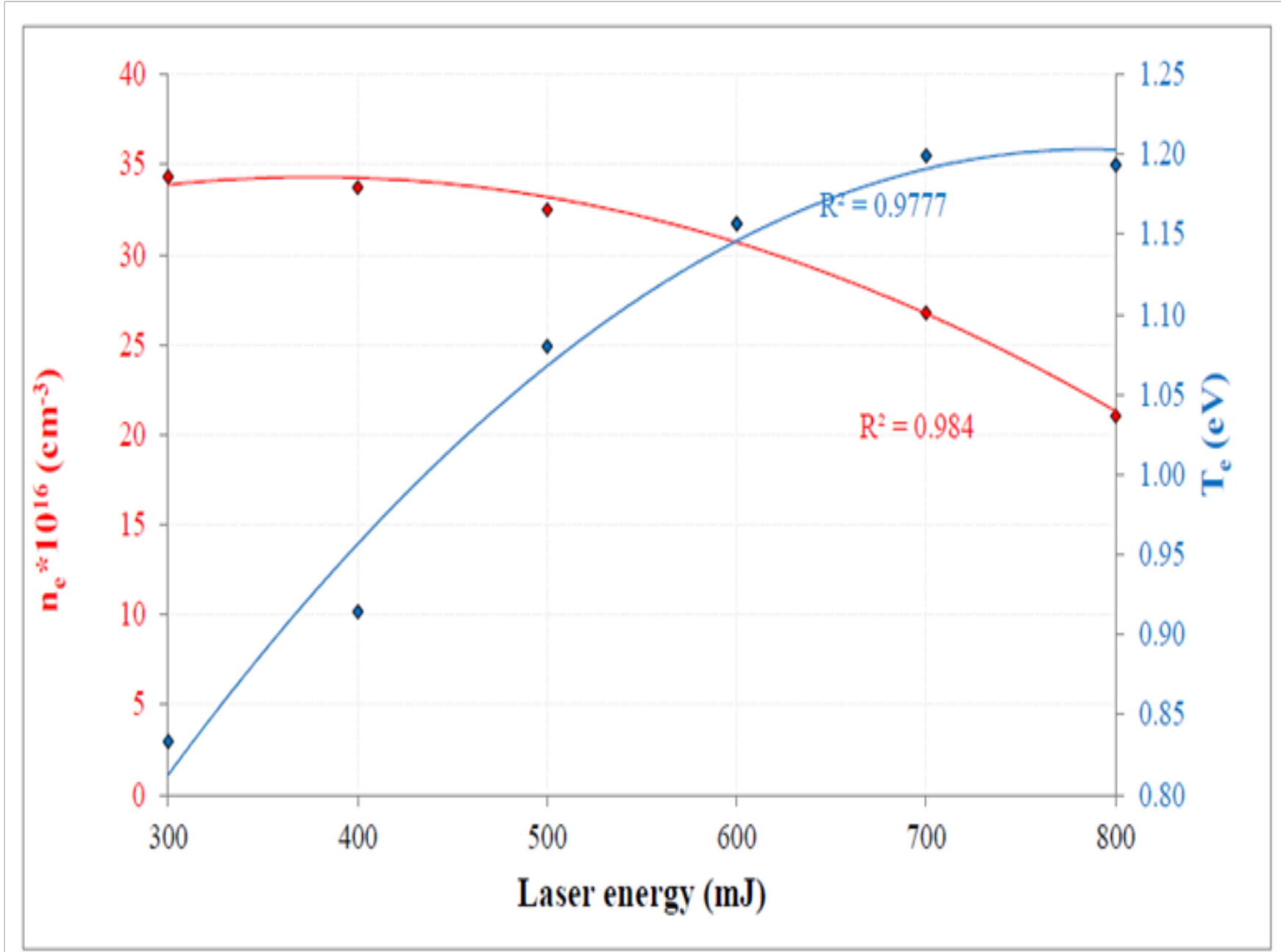

Figure 4: The variation of (Te) and (ne) versus the laser energy for Si: Al alloy in air 


\section{References}

1) Spatschek KH (2008) Introduction to theoretical plasma physics.

2) Keloth A (2012) Laser Induced Breakdown Spectroscopy (LIBS) and time resolved studies of copper plasma excited by nanosecond and femtosecond laser pulses. Cochin University of Science and Technology, M.Sc Project report, 2012.

3) Jiang X (2012) Dual-pulse laser induced breakdown spectroscopy in the vacuum ultraviolet with ambient gas: spectroscopic analysis and optimization of limit of detection of carbon and sulfur in steel. Dublin City University.

4) Vickers JD, Winefordner TJ (1978) In analytical spectroscopy series, Part 2, Chapter 7. E.L. Grove 1.

5) Barnes R (1978) CRC Crit Rev Anal Chem 9: 203-296.

6) Boumans PWJM (1972) Analytical Spectroscopy Series, Part 2, Chapter 6. E.L. Grove 1

7) Walters J (1977) Science 198: 787-797.

8) Tsuda N, Yamaguchi T, Yamada J (2009) Property of laser-induced plasma in liquid. J Plasma Fusion Res 8: 619-622.

9) Ley HH (2014) Analytical Methods in Plasma Diagnostic by Optical Emission Spectroscopy : A Tutorial Review J Sci Technol 6: 49-66.

10) National Institute of Standards and Technology (NIST) Atomic spectra database, version 5 .

11) Hussain AA, Al-Razzaq AA (2016) Plasma characteristics of Ag: Al alloy produced by fundamental and second harmonic frequencies of $\mathrm{Nd}$ : YAG laser. Iraqi Journal of Physics 14: 205-214.

Submit your manuscript to a JScholar journal and benefit from:

I Convenient online submission

ब Rigorous peer review

I Immediate publication on acceptance

- Open access: articles freely available online

9 High visibility within the field

I Better discount for your subsequent articles

Submit your manuscript at http://www.jscholaronline.org/submit-manuscript.php 